\title{
Rearrangement of cluster structure during fission processes
}

\author{
A G Lyalin $\uparrow+$, O I Obolensky§, A V Solov’yov $§$, Il A \\ Solov'yov $\$$ and W Greiner $\$$ \\ $\dagger$ Institute of Physics, St Petersburg State University, Ulianovskaya str. 1, 198504 St \\ Petersburg, Petrodvorez, Russia \\ $\ddagger$ Institut für Theoretische Physik der Universität Frankfurt am Main, Robert-Mayer \\ Str. 8-10, D-60054 Frankfurt am Main, Germany \\ $\S$ A.F. Ioffe Physical-Technical Institute, Russian Academy of Sciences, \\ Politechnicheskaja str. 26, 194021 St Petersburg, Russia \\ E-mail: lyalin@th.physik.uni-frankfurt.de
}

\begin{abstract}
Results of molecular dynamics simulations of fission reactions $\mathrm{Na}_{10}^{2+} \rightarrow$ $N a_{7}^{+}+N a_{3}^{+}$and $N a_{18}^{2+} \rightarrow 2 N a_{9}^{+}$are presented. Dependence of the fission barriers on isomer structure of the parent cluster is analyzed. It is demonstrated that the energy necessary for removing homothetic groups of atoms from the parent cluster is largely independent of the isomer form of the parent cluster. Importance of rearrangement of the cluster structure during the fission process is elucidated. This rearrangement may include transition to another isomer state of the parent cluster before actual separation of the daughter fragments begins and/or forming a "neck" between the separating fragments.
\end{abstract}


Fission of charged atomic clusters occurs when repulsive Coulomb forces, arising due to the excessive charge, overcome the electronic binding energy of the cluster [1, 2, 3]. This mechanism of the cluster fission is in a great deal similar to the nuclear fission phenomena. Experimentally, multiply charged metal clusters can be observed in the mass spectra when their size exceeds the critical size of stability, which depends on the metal species and cluster charge [4, 5, 6].

We report the results of the ab initio molecular dynamics (MD) simulations of the fission processes $N a_{10}^{2+} \rightarrow N a_{7}^{+}+N a_{3}^{+}$and $N a_{18}^{2+} \rightarrow 2 N a_{9}^{+}$. Both symmetric and asymmetric fission channels are considered. We have investigated the parent cluster isomer dependence of the fission barrier for the reaction $N a_{10}^{2+} \rightarrow N a_{7}^{+}+N a_{3}^{+}$. To the best of our knowledge, a comparative study of fission barriers for various isomers by means of quantum chemistry methods has not been carried out before. Note that such a study is beyond the scope of simpler approaches which do not account for ionic structure of a cluster.

We found that the direct separation barrier for the reaction $N a_{10}^{2+} \rightarrow N a_{7}^{+}+N a_{3}^{+}$ has a weak dependence on the isomeric structure of the parent cluster. We note, however, that the groups of atoms to be removed from the parent cluster isomers must be chosen with care; one has to identify homothetic groups of atoms in each fissioning isomer. The weak dependence on the isomeric state of the parent $N a_{10}^{2+}$ cluster implies that the particular ionic structure of the cluster is largely insignificant for the shape and height of the fission barrier. This is due to the fact that the maximum of fission barriers in considered cases are located at distances comparable or exceeding the sum of the resulting fragments radii. At such distances the interaction between the fragments, apart from the Coulombic repulsion, is mainly determined by the electronic properties rather than by the details of the ionic structure of the fragments. This is an important argument for justification of the jellium model approach to the description of the fission process of multiply charged metal clusters.

We have demonstrated the importance of rearrangement of the cluster ionic structure during the fission process. The possibility of rearrangement of the cluster structure leads to the fact that direct fission of a cluster isomer in some cases may not be the energetically optimum path for the fission reaction. Alternatively, the reaction can go through transition to another isomer state of the parent cluster. This transition can occur in the first phase of the fission process, before separation of the fragments actually begins. We show that this is the case for the fission of $C_{4 v}$ and $D_{4 d}$ isomers of $N a_{10}^{2+}$ cluster.

The rearrangement of ionic structure may be important also after the fragments began to separate. For $N a_{18}^{2+} \rightarrow 2 N a_{9}^{+}$reaction, two magic fragments $N a_{9}^{+}$form a metastable transitional state in which the fragments are connected by a "neck". This "necking" allows for significant reduction in the height of the fission barrier. Note that the similar necking phenomenon is known for the nuclear fission process [7].

In our molecular dynamics simulations we utilize methods of density functional 
theory (DFT). Within the DFT one has to solve the Kohn-Sham equations [8]

$$
\left(\frac{p^{2}}{2}+U_{\mathrm{i}}+V_{\mathrm{H}}+V_{\mathrm{xc}}\right) \psi_{i}=\varepsilon_{i} \psi_{i}
$$

where the first term corresponds to the kinetic energy of an electron from the KohnSham reference system, $U_{\mathrm{i}}$ describes the attraction of the $i^{\text {th }}$ electron to the nuclei in the cluster, $\psi_{\mathrm{i}}$ is the electronic orbital, $V_{\mathrm{H}}$ is the Hartree part of the inter-electronic interaction,

$$
V_{\mathrm{H}}(\mathbf{r})=\int \frac{\rho\left(\mathbf{r}^{\prime}\right)}{\left|\mathbf{r}-\mathbf{r}^{\prime}\right|} \mathrm{d} \mathbf{r}^{\prime}
$$

$\rho(\mathbf{r})$ is the electron density, $V_{\mathrm{xc}}$ is the local exchange-correlation potential defined as the functional derivative of the exchange-correlation energy functional

$$
V_{\mathrm{xc}}=\frac{\delta E_{\mathrm{xc}}[\rho]}{\delta \rho(\mathbf{r})}
$$

where the exchange-correlation energy is partitioned into two parts, referred to as exchange and correlation parts:

$$
E_{\mathrm{xc}}[\rho]=E_{\mathrm{x}}(\rho)+E_{\mathrm{c}}(\rho) .
$$

Physically, these two terms correspond to same-spin and mixed-spin interactions, respectively. Both parts are functionals of the electron density, which can be of two distinct types: either a local functional depending on the electron density $\rho$ only or a gradient-corrected functional depending on the electron density and its gradient $\nabla \rho$.

There is a variety of exchange-correlation functionals in the literature. We have used the three-parameter Becke-type gradient-corrected exchange functional with the gradient-corrected correlation functional of Lee, Yang, and Parr (B3LYP) 9]. For the explicit form of this functional we refer to the original papers [10, 11, 12, The B3LYP functional has proved to be a reliable tool for studying the structure and properties of small metal clusters. It provides high accuracy at comparatively low computational costs. For a discussion and a comparison with other approaches, see [13, 14]. Note that the density of the parent cluster and two daughter fragments (including the overlapping region before scission point) almost does not change during the fission process (by analogy with the deformed jellium model, see [15, 16] for more details). This means that the B3LYP method works adequately for any fragment separation distances, $d$, during the fission process.

The calculations have been carried out with the use of the GAUSSIAN 98 software package [17. The 6-311G(d) and LANL2DZ basis sets of primitive Gaussian functions have been used to expand the cluster orbitals [18. The 6-311G(d) basis has been used for simulations involving $N a_{10}^{2+}$ cluster. This basis set takes into account electrons from all atomic orbitals, so that the dynamics of all particles in the system is taken into account. For $N a_{18}^{2+}$ cluster we have used more numerically efficient LANL2DZ basis, for which valent atomic electrons move in an effective core potential (see details in [18]).

To simulate the fission process we start from the optimized geometry of a cluster (for details of the geometry optimization procedure see [13, 14]) and choose the atoms 
the resulting fragments would consist of. The atoms chosen for a smaller fragment are shifted from their locations in the parent cluster to a certain distance. Then, the multidimensional potential energy surface, its gradient and forces with respect to the molecular coordinates are calculated. These quantities specify the direction along the surface in which the energy decreases the most rapidly and provide information for the determination of the next step for the moving atoms. If the fragments are removed not far enough from each other then the attractive forces prevailed over the repulsive ones and the fragments stuck together forming the unified cluster again. In the opposite situation the repulsive forces dominate and the fragments drift away from each other. The dependence of the total energy of the system on the fragment separation distance forms the fission barrier. The aim of our simulations is to find the fission pathway corresponding to the minimum of the fission barrier.

There are usually many stable isomers of a cluster with energies slightly exceeding the energy of the ground state isomer. In order to analyze the isomer dependence of the fission barrier in the reaction $N a_{10}^{2+} \rightarrow N a_{7}^{+}+N a_{3}^{+}$we have picked two energetically lowlying isomers with the point symmetry groups $C_{4 v}$ and $D_{4 d}$ differing from the distorted $T_{d}$ point symmetry group of the ground state parent $N a_{10}^{2+}$ cluster. Three isomer states of the $N a_{10}^{2+}$ cluster are shown in figure 1.
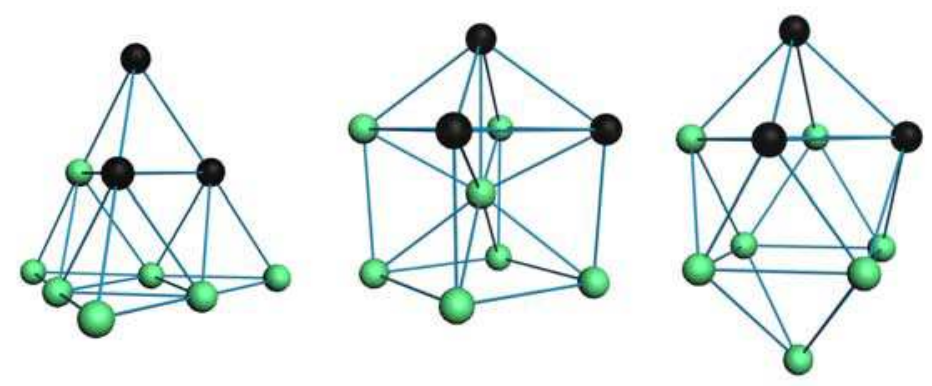

Figure 1. Three isomers of $N a_{10}^{2+}$ cluster. From left to right: the ground state isomer of distorted $T_{d}$ point symmetry group (total energy is -1622.7063 a.u.); an isomer of $C_{4 v}$ point symmetry group (total energy is -1622.6888 a.u., that exceeds the lowest energy state by $0.476 \mathrm{eV}$ ); an isomer of $D_{4 d}$ point symmetry group (total energy is -1622.6860 a.u., that exceeds the lowest energy state by $0.553 \mathrm{eV}$ ). The homothetic group of three atoms marked by black color.

In figure 2 we show fission barriers for separation three atoms from the $C_{4 v}, D_{4 d}$, and $T_{d}$ isomers of the $N a_{10}^{2+}$ cluster. In this figure zero level of energy is chosen for each parent isomer separately and corresponds to the minimum of total energy of that isomer. The initial distances between the centers of mass of two (future) fragments are finite so that the barriers do not start at the origin.

The barriers for all three channels are close. The weak sensitivity of the fission barrier on the isomeric states of the reactants can be explained if one notices that the barrier maxima are located at distances comparable to or exceeding the sum of the 


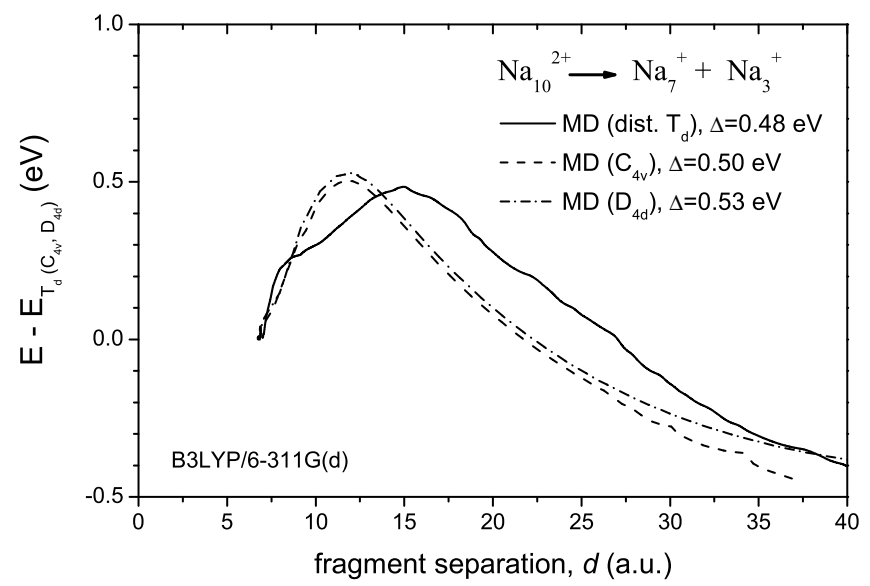

Figure 2. Fission barriers for separating the homothetic group of three atoms (marked by black color in figure 10 from three isomers of $N a_{10}^{2+}$ cluster derived from molecular dynamics simulations (direct $N a_{10}^{2+} \rightarrow N a_{7}^{+}+N a_{3}^{+}$fission channel). The barriers plotted versus distance between the centers of mass of the fragments. Solid, dashed, and dashed-dotted lines correspond to distorted $T_{d}, C_{4 v}$, and $D_{4 d}$ point symmetry groups isomers of the parent cluster, respectively. Energies are measured from the energy of the ground state of the corresponding isomers, i.e. we plot $E-E_{T_{d}\left(C_{4 v}, D_{4 d}\right)}$, where $E$ is the total energy of the system and $E_{T_{d}\left(C_{4 v}, D_{4 d}\right)}$ are the ground energies of the $T_{d}, C_{4 v}$ and $D_{4 d}$ isomer states of the parent $N a_{10}^{2+}$ cluster, respectively.

resulting fragments radii, that is not far from the scission point. At such distances the interaction between the fragments, apart from Coulombic repulsion, is mainly determined by the electronic properties rather than by the details of the ionic structure of the fragments. This is an important argument for justification of the jellium model approach to the description of the fission process of multiply charged metal clusters.

It is important to note that the barriers presented in figure 2 are calculated in assumption that fission occurs for the fixed (given) isomers. However, since $C_{4 v}$ and $D_{4 d}$ isomers are not the lowest energy states of $N a_{10}^{2+}$ system, there could be other processes competing with fission. One of such processes is rearrangement of the cluster structure.

Rearrangement of the cluster structure during the fission process may significantly reduce the fission barrier. Such rearrangement may occur before the actual separation of the daughter fragments begins or after that.

Fission of $C_{4 v}$ and $D_{4 d}$ isomers of $N a_{10}^{2+}$ cluster is an example of situation where rearrangement of the cluster structure takes place before the fragments start to separate. In figure 3 we show schematically the total energies of the $N a_{10}^{2+} T_{d}, C_{4 v}$ and $D_{4 d}$ isomers and barriers for the transitions between those states. It is seen from the figure that transition to the ground $\left(T_{d}\right)$ state with subsequent fission into the $N a_{3}^{+}$and $N a_{7}^{+}$ fragments, $N a_{10}^{2+}\left(C_{4 v}\right.$ or $\left.D_{4 d}\right) \rightarrow N a_{10}^{2+}\left(T_{d}\right) \rightarrow N a_{7}^{+}+N a_{3}^{+}$, (shown by solid lines) is 


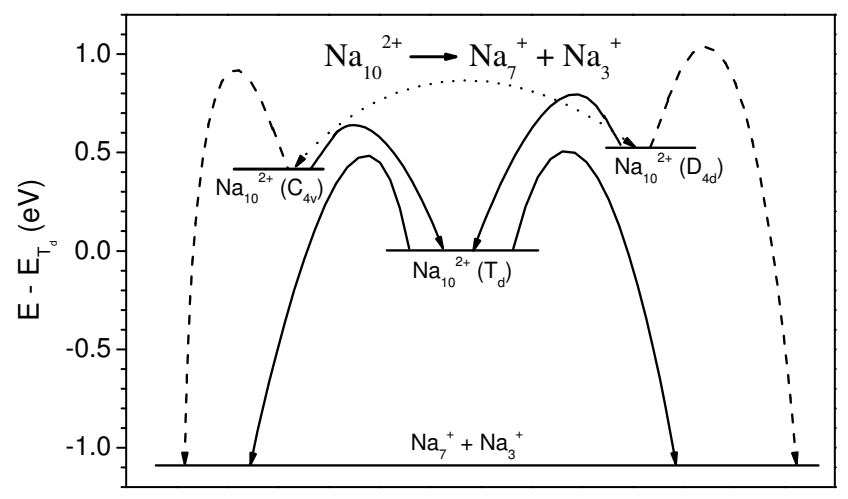

Figure 3. Energy levels of some states of the $N a_{10}^{2+}$ system and schematic barriers for transitions between these states. Energies are measured from the energy of the ground $T_{d}$ state of the $N a_{10}^{2+}$ cluster.

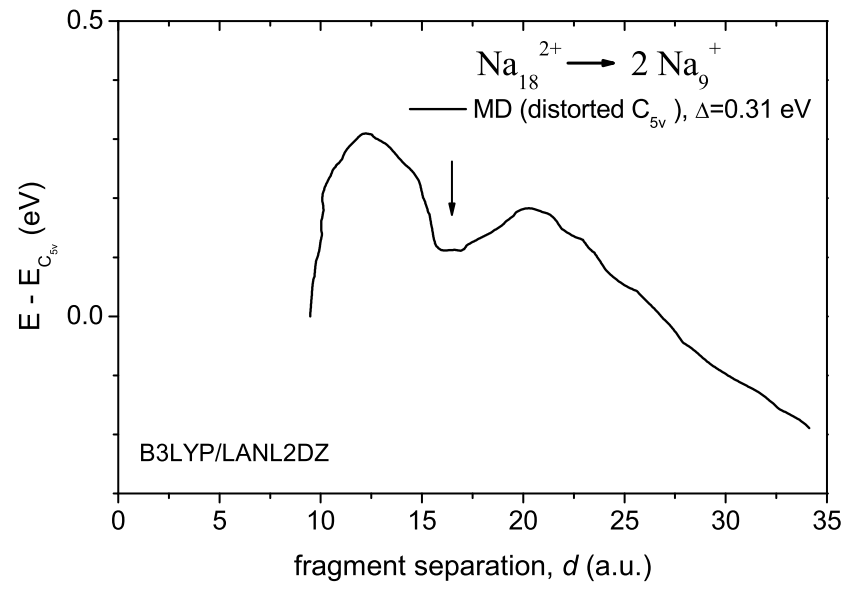

Figure 4. Fission barrier for $N a_{18}^{2+} \rightarrow 2 N a_{9}^{+}$channel derived from molecular dynamics simulations as a function of distance between the centers of mass of the fragments. Energy is measured from the energy of the ground $C_{5 v}$ state of the $\mathrm{Na}_{18}^{2+}$ cluster. The arrow shows position of the meta-stable transitional state, see also figure 5 This results is in a good agreement with the results of the jellium model [15] 16].

preferred path for fission of both $C_{4 v}$ and $D_{4 d}$ isomers of the $N a_{10}^{2+}$ cluster and requires only about $0.2 \mathrm{eV}$ for $C_{4 v}$ isomer and $0.26 \mathrm{eV}$ for $D_{4 d}$ isomer. In contrast, the direct fission process, $N a_{10}^{2+}\left(C_{4 v}\right.$ or $\left.\mathrm{D}_{4 \mathrm{~d}}\right) \rightarrow \mathrm{Na}_{7}^{+}+\mathrm{Na}_{3}^{+}$, (shown by dashed lines) requires about $0.5 \mathrm{eV}$. We also show the barrier for the transition between the $C_{4 v}$ and $D_{4 d}$ isomers.

Another example of cluster structure rearrangement in the fission process is the $N a_{18}^{2+} \rightarrow 2 N a_{9}^{+}$reaction. The fission barrier for this reaction is shown in figure 4 If 


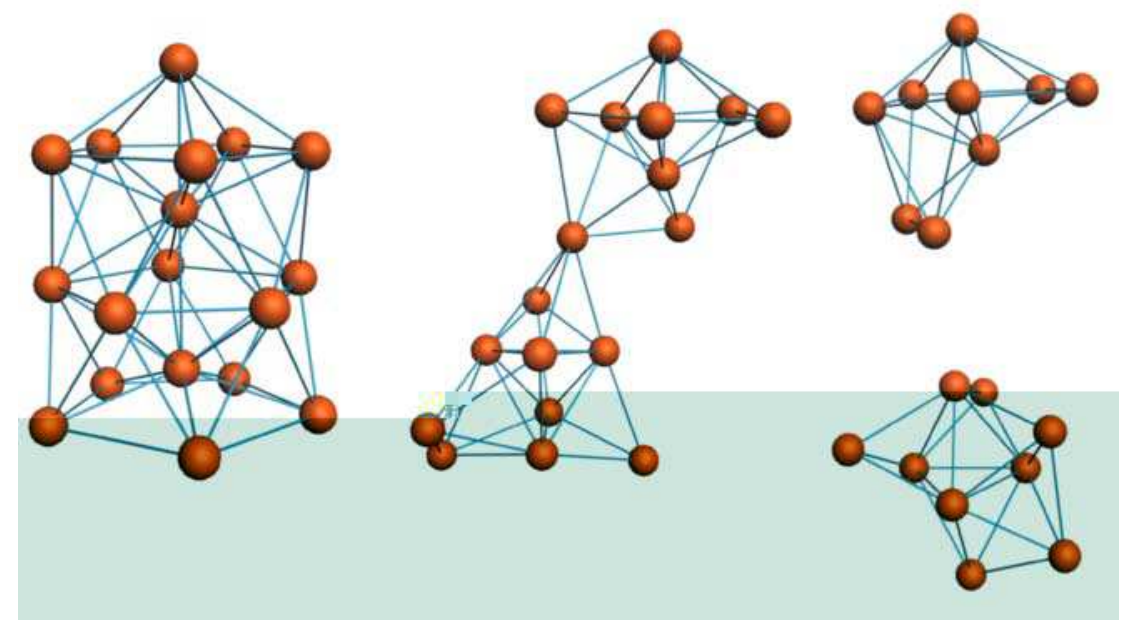

Figure 5. Rearrangement of the cluster structure during the fission process $N a_{18}^{2+} \rightarrow$ $2 N a_{9}^{+}$. From left to right: ground state of the parent cluster; "necking" between the two fragments leads to a meta stable intermediate state and significantly reduces the fission barrier height; two $N a_{9}^{+}$fragments drifting away from each other.

Table 1. Summary of the fission barrier heights (eV).

\begin{tabular}{lll}
\hline & $N a_{10}^{2+} \rightarrow N a_{7}^{+}+N a_{3}^{+}$ & $N a_{18}^{2+} \rightarrow 2 N a_{9}^{+}$ \\
\hline MD (this work) & 0.49 (distorted $\left.T_{d}\right)$ & 0.31 \\
MD[19] & 0.67 & 0.52 \\
MD[20] & 0.54 & - \\
Jellium model[15] 16] & 0.16 & 0.48 \\
\hline
\end{tabular}

two fragments of the parent cluster were not allowed to adjust their ionic structure the fission barrier would be about $1 \mathrm{eV}$. Rearrangement of the cluster structure allows to reduce the fission barrier down to $0.31 \mathrm{eV}$. During the fission process the daughter fragments start to drift away from each other and a "neck" forms between the fragments. Formation of the "neck" results in a metastable transitional state. The geometry of this state, as well as the geometry of the parent cluster are shown in figure 5 .

In table 1 we have summarized our results for the fission barrier heights and compared them with the results of other molecular dynamics simulations and with the predictions of the jellium model.

We have investigated two aspects of charged metal cluster fission process: dependence of the fission barrier on isomer state of the parent cluster and importance of rearrangement of the cluster ionic structure during the fission process.

We found that for a consistent choice of the atoms removed from the cluster the fission barrier for the reaction $N a_{10}^{2+} \rightarrow N a_{7}^{+}+N a_{3}^{+}$has a weak dependence on the initial isomer structure of the parent cluster. This implies that the particular ionic structure of the cluster is largely insignificant for the height of the fission barrier. This is an important argument for justification of the jellium model approach to the description 
of the fission process of multiply charged metal clusters.

We have shown importance of rearrangement of the cluster ionic structure during the fission process. The fission reaction can go through transition to another isomer state of the parent cluster. This transition can occur before actual separation of the fragments begins and/or "neck" between the separating fragments is formed. In any case the resulting fission barrier can be significantly lower compared to the one for the direct fission path.

\section{Acknowledgments}

The authors acknowledge support of this work by the Alexander von Humboldt Foundation, DFG, the Studienstiftung des deutschen Volkes, INTAS, Russian Foundation for Basic Research (grant No 03-02-16415-a), Russian Academy of Sciences (grant 44) and the Royal Society of London.

\section{References}

[1] Sattler K, Mühlbach J, Echt O, Pfau P and Recknagel E 1981 Phys. Rev. Lett. 47160

[2] Näher U, Bjornholm S, Frauendorf F and Guet C 1997 Phys. Rep. 285245

[3] Yannouleas C, Landman U and Barnett R N 1999 in Metal Clusters, edited by W. Ekardt (Wiley, New York) p 145

[4] Bréchignac C, Cahuzac Ph, Carlier F and Leygnier J 1989 Phys. Rev. Lett. 631368

[5] Bréchignac C, Cahuzac Ph, Carlier F and de Frutos M 1994 Phys. Rev. B 492825

[6] Martin T P 1984 J. Chem. Phys. 814426

[7] Eisenberg J M and Greiner W 1985 Nuclear Theory. vol 1. Collective and Particle Models, (North Holland, Amsterdam).

[8] Kohn W and Sham L J 1965 Phys. Rev. A 1401133

[9] Becke A D 1993 J. Chem. Phys. 985648

[10] Becke A D 1988 Phys. Rev. A 383098

[11] Vosko S H, Wilk L and Nusair M 1980 Can. J. Phys. 581200

[12] Lee C, Yang W and Parr R G 1988 Phys. Rev. B 37785

[13] Solov'yov Il A, Solov'yov A V and Greiner W 2002 Phys. Rev. A 65053203

[14] Lyalin A G, Solov'yov Il A, Solov'yov A V and Greiner W 2003 Phys. Rev. A 67063203

[15] Lyalin A, Solov'yov A, Greiner W and Semenov S 2002 Phys. Rev. A 65023201

[16] Lyalin A, Solov'yov A and Greiner W 2002 Phys. Rev. A 65043202

[17] Frisch M J et al 1998 computer code GAUSSIAN 98, Rev. A. 11 (Gaussian Inc., Pittsburgh, PA)

[18] Foresman J B and Aeleen Frisch 1996 Exploring Chemistry with Electronic Structure Methods (Gaussian Inc., Pittsburgh, PA)

[19] Montag B, Reinhard P G 1995 Phys. Rev. B 5216365

[20] Blaise B, Blundell S A, Guet C and Zope Rajendra R 2001 Phys. Rev. Lett. 87063401 